\title{
Poder de pesca, esfuerzo de pesca y cambios estacionales e interanuales en la abundancia relativa de Strangomera bentincki y Engraulis ringens en el área frente a Talcahuano, Chile (1990-97) *
}

\author{
Luis Cubillos S., Mariella Canales, Aldo Hernández R., Doris Bucarey S., \\ Lidia Vilugrón y Leonardo Miranda A. \\ Instituto de Investigación Pesquera \\ Casilla 350, Talcahuano, Chile. E-mail: inpesca@arauco.reuna.cl
}

\begin{abstract}
RESUMEN. Se analiza el poder de pesca de la flota de cerco que orienta su esfuerzo hacia la sardina común (Strangomera bentincki) y anchoveta (Engraulis ringens) en el área de Talcahuano $\left(37^{\circ} \mathrm{S}-73^{\circ} \mathrm{W}\right)$ durante el período $1990-97$. Se estandariza el esfuerzo de pesca y se analizan los cambios estacionales e interanuales de la captura por unidad de esfuerzo (CPUE). Las tasas de captura se analizan mediante un modelo lineal generalizado, el cual considera como variable independiente la capacidad de bodega (CB) de las embarcaciones y como factores los años y meses, además de un factor denominado período que toma en cuenta la época invernal (abril-septiembre) y la estival (octubre-marzo). Se utiliza el concepto de poder de pesca relativo para estandarizar las tasas de captura, utilizando como estándar el estrato 40-59 $\mathrm{m}^{3}$ de CB. Las tasas de captura mensuales se relacionan significativamente con la CB y con varias combinaciones de interacción entre los factores. La estacionalidad de la pesquería se observa con valores máximos en las capturas, esfuerzo de pesca y abundancia relativa durante la época estival. Este comportamiento estacional se debe al reclutamiento de $S$. bentincki que ocurre en noviembre-diciembre, a las mejores condiciones climáticas de la época que facilitan la operación de la flota y a la reorientación temporal del esfuerzo de pesca de una fracción de la flota industrial que extrae jurel (Trachurus symmetricus murphyi) en invierno. Interanualmente el esfuerzo de pesca estándar no presenta tendencias significativas, pero se observan cambios en la abundancia relativa de ambos recursos. La CPUE de $S$. bentincki declina desde comienzos de 1991 hasta mediados de 1995, recuperándose en 1996-97. En cambio, la CPUE de E. ringens, fue mayor en el período 1993-94, declina en 1995 y se estabiliza en 1996-97.
\end{abstract}

Palabras claves: esfuerzo de pesca, abundancia relativa, sardina común, anchoveta, Talcahuano, Chile.

\section{Fishing power, fishing effort and seasonal and interannual changes in the relative abundance of Strangomera bentincki and Engraulis ringens in the area off Talcahuano, Chile (1990-97)*}

\begin{abstract}
An analysis of the fishing power of the purse-seine fleet fishing for common sardine (Strangomera bentincki) and anchovy (Engraulis ringens) in the area off Talcahuano $\left(37^{\circ} \mathrm{S}-73^{\circ} \mathrm{W}\right)$, during the period $1990-97$, is carried out. Fishing effort is standardized and seasonal and interannual changes in the catch per unit effort (CPUE) are analyzed. Catch rates are analyzed considering the hold capacity (HC) of the ships as an independent variable, the years and months as factors, and also it was used a factor denominated period that takes into account the winter time (April-September) and summery (October-March). In order to standardize the catch rates the relative fishing power was used considering the stratum of $40-59 \mathrm{~m}^{3} \mathrm{HC}$ as a standard. Monthly catch rates are related significantly to the $\mathrm{HC}$ of the ships and to the interactions among the factors. The fishery shows a seasonal behavior where the maximum values of the catches, fishing effort and CPUE are observed during spring and summer time. This seasonal behavior is due to the recruitment of $S$. bentincki that happens in November-December, to the best climatic conditions to fish during spring and summer, and also to the partial reorientation of the fishing effort of a fraction of the industrial fleet that fishes jack mackerel (Trachurus symmetricus murphyi) in winter time. Annually, the standard fishing effort doesn't present significant tendencies, but changes in the indexes of relative abundance of both resources are observed. The CPUE of S. bentincki declines from beginnings of 1991 until half of 1995 and the CPUE recovers in 1996 and 1997. The E. ringens CPUE, on the other hand, was high in the period 1993-94, it declines in 1995 and it is stabilized in 1996-97.
\end{abstract}

Key words: fishing effort, relative abundance, common sardine, anchovy, Talcahuano, Chile.

* Proyectos del Fondo de Investigación Pesquera N 94-11 y N 96-10. 


\section{INTRODUCCION}

En la zona centro-sur de Chile $\left(33^{\circ} \mathrm{S}-39^{\circ} \mathrm{S}\right)$ se desarrolla una importante actividad pesquera industrial y artesanal, desembarcándose principalmente jurel, Trachurus symmetricus murphyi (Nichols), y secundariamente sardina común, Strangomera bentincki (Norman 1936), y anchoveta, Engraulis ringens (Jenyns 1842). Aunque la pesquería de T. s. murphyi es la más importante, con niveles de captura que alcanzaron a 3,5 millones de toneladas en 1996, los desembarques de $S$. bentincki y E. ringens en la zona han presentado siempre un comportamiento fluctuante. En 1989, por primera vez, el desembarque de estos dos recursos supera los niveles máximos registrados en la historia de la pesquería, manteniéndose en la década de los años 90 por sobre las 400 mil toneladas anuales y lográndose desembarques máximos en 1991, 1994 y 1996 (Fig. 1). Se destaca que la flota pesquera que orienta el esfuerzo de pesca hacia $S$. bentincki y E. ringens es diferente de la flota que se dedica a la extracción de T. s. murphyi. La flota que opera sobre los clupeiformes está compuesta por cerca de 230 embarcaciones, de las cuales más del $50 \%$ tienen una capacidad de bodega menor a $80 \mathrm{~m}^{3}$ (Cubillos y Arancibia, 1993a, b).

Varios estudios se han realizado sobre estos recursos y su pesquería, destacando aquellos que des-

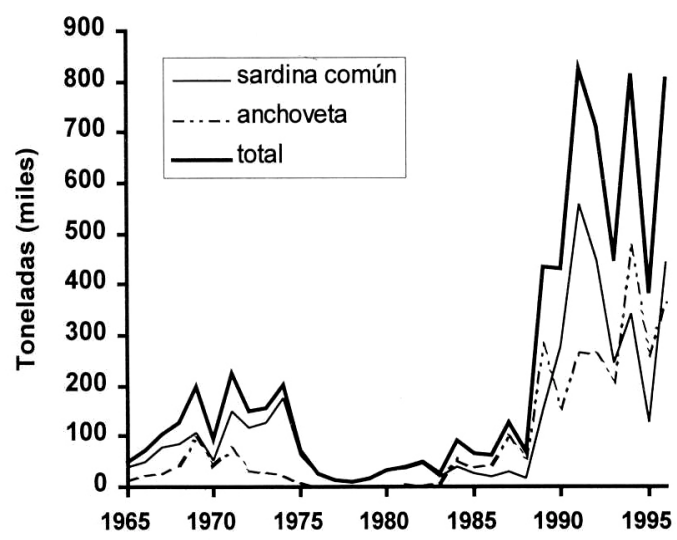

Figura 1. Desembarque anual de sardina común y anchoveta en la zona centro-sur de Chile. Fuente: Servicio Agrícola y Ganadero, división de Pesca y Caza (1965-77) y Servicio Nacional de Pesca (1978-96).

Figure 1. Annual landing of common sardine and anchovy in central-southern Chile. Source: Servicio Agricola y Ganadero, división de Pesca y Caza (196577) y Servicio Nacional de Pesca (1978-96). criben la situación del recurso antes de 1977, cuando constituían el principal recurso pesquero de la zona (Serra, 1978, 1983; Guerrero y Yáñez, 1986), y aquellos que analizan los cambios de la abundancia en relación con la variabilidad ambiental (Fonseca et al., 1986; Yáñez et al., 1990, 1992), entre otros. Recientemente, Cubillos y Arancibia (1993a, b) analizan los principales aspectos biológicos y pesqueros de S. bentincki y E. ringens en 1990 y 1991, cuando estas especies nuevamente pasan a constituirse como recursos de importancia en el desembarque (Fig. 1), y alternativos a T. s. murphyi, particularmente en primavera cuando las capturas de este último recurso disminuyen.

El propósito de este trabajo es analizar el poder de pesca de la flota, estandarizar el esfuerzo de pesca y caracterizar los cambios estacionales e interanuales de la abundancia de $S$. bentincki y $E$. ringens ocurridos durante el período 1990-97, utilizando como índice de abundancia la captura por unidad de esfuerzo estándar.

\section{MATERIALES Y METODOS}

\section{Area de estudio y fuente de información}

El área de estudio corresponde a aquella donde opera preferentemente la flota artesanal e industrial que dirige su esfuerzo de pesca hacia $S$. bentincki y $E$. ringens, cuya extensión se puede considerar entre los $34^{\circ} 30^{\prime} \mathrm{S}$ y $39^{\circ} \mathrm{S}$ en la zona centro-sur de Chile.

Se utiliza información de capturas mensuales en peso (toneladas) desembarcadas por la flota sardinera en Talcahuano, San Vicente, Coronel y Lota, así como la actividad de cada embarcación que ha participado en la pesquería en el período comprendido entre enero de 1990 y julio de 1997. Esta información fue obtenida de los registros diarios que llevan las empresas pesqueras en la VIII Región del país, la que fue recopilada directamente por personal del Instituto de Investigación Pesquera (INPESCA). Esta información fue digitada y procesada utilizando planillas electrónicas y programas computacionales que permiten conformar una base de datos ad hoc.

En los registros de las empresas pesqueras se detalla la operación y descarga de cada embarcación, permitiendo conocer la captura total por especie y el esfuerzo de pesca desarrollado en términos de viajes con pesca. Otras medidas de esfuerzo, tal como los viajes totales o los días fuera de puerto, 
no están disponibles para las embarcaciones menores a $100 \mathrm{~m}^{3}$ de capacidad de bodega. Así, la cuantificación de viajes con pesca fue la única medida disponible para cuantificar el tiempo de operación de la flota que actúa sobre $S$. bentincki y $E$. ringens.

Las capturas utilizadas se basan entonces en los registros que llevan las empresas pesqueras por embarcaciones y no en la estadística oficial de desembarque que publica el Servicio Nacional de Pesca. Las capturas por especie se determinan utilizando la proporción relativa en peso de cada especie, cuya información es obtenida rutinariamente por el personal del INPESCA, a partir de muestreos diarios de proporción de especie y de frecuencia de tamaños en las capturas de estos clupeiformes en los principales puertos de la VIII Región (Talcahuano, San Vicente, Coronel y Tomé).

\section{Estandarización del esfuerzo de pesca}

Las tasas de captura (captura por tiempo de operación) de cada unidad de pesca pueden variar debido a diferentes causas; entre ellas, las fluctuaciones de la abundancia, de la disponibilidad y de la capturabilidad del recurso, y de las condiciones climáticas y oceanográficas (Yáñez y Maritano, 1983). Por lo tanto, el poder de pesca absoluto de cada embarcación usualmente es imposible de determinar, razón por la cual se utiliza el concepto de poder de pesca relativo (PPR), definido como la razón entre la tasa de captura de cada unidad de pesca respecto de la tasa de captura de otras embarcaciones consideradas como patrón (Shimada y Shaefer, 1956; Kimura, 1981):

$$
\operatorname{PPR}_{\mathrm{i}}=\frac{\mathrm{R}_{\mathrm{i}}}{\mathrm{R}^{*}}
$$

donde $\mathrm{PPR}_{\mathrm{i}}$ es el poder de pesca relativo del barco $\mathrm{i}, \mathrm{R}_{\mathrm{i}}$ es la tasa de captura del barco i (viajes con pesca) y $R^{*}$ es la tasa de captura del barco estándar.

Luego, el esfuerzo de pesca estándar $\left(\mathrm{E}^{*}{ }_{\mathrm{i}, \mathrm{t}}\right)$ del barco i durante el período $\mathrm{t}$ (mes), corresponde al producto entre el esfuerzo de pesca nominal $\left(\mathrm{E}_{\mathrm{it}}\right)$ y el poder de pesca relativo del barco i:

$$
\mathrm{E}_{\mathrm{i}, \mathrm{t}}^{*}=\mathrm{E}_{\mathrm{it}} \mathrm{PPR}_{\mathrm{i}}
$$

\section{Análisis de las tasas de captura}

Los factores que condicionan el poder de pesca son de naturaleza variada, tal como las características físicas de cada unidad de pesca, de las artes de pesca, de la tripulación, del comportamiento, disponibilidad y vulnerabilidad del stock, así como de los cambios asociados al hidroclima (Kimura, 1981; Yáñez y Maritano, 1983). Sin embargo, usualmente son las características físicas de las unidades de pesca las que mejor se relacionan con el poder de pesca (Carlson, 1975; Kimura 1981), particularmente el tamaño expresado a través de la capacidad de bodega (CB), ya que esta variable se correlaciona significativamente con otras variables geométricas y funcionales de las embarcaciones (Shimada y Schaefer, 1956). Con el objeto de analizar la incidencia de la CB sobre las tasas de captura anuales de cada embarcación, se utilizó el siguiente modelo de regresión:

$$
\ln \left(\mathrm{R}_{\mathrm{i}}\right)=\ln \left(\frac{\mathrm{C}_{\mathrm{i}}}{\mathrm{VCP}_{\mathrm{i}}}\right)=\ln (\mathrm{a})+\mathrm{b} \ln \left(\mathrm{CB}_{\mathrm{i}}\right)+\mathrm{e}
$$

donde el subíndice i denota la i-ésima embarcación, $\mathrm{R}$ es la tasa de captura anual (captura por tiempo de operación), C es la captura anual, VCP son los viajes con pesca acumulados en el año, a y b son las constantes de la regresión, y e es una variable error con promedio cero y varianza constante.

No obstante, debido a los cambios estacionales propios en esta pesquería, tanto en lo que a la composición de la flota concierne como a la estacionalidad de los rendimientos, del esfuerzo de pesca y de las capturas (Cubillos y Arancibia, 1993a, b), se utilizó un modelo lineal generalizado para analizar las tasas de captura (Allen y Punsly, 1984; Hilborn y Walters, 1992):

$\ln (\mathrm{R})=\mathrm{m}+\mathrm{a}+\mathrm{b}+\mathrm{c}+\mathrm{CB}+$ interacciones $+\mathrm{e}$

donde $\mathrm{m}$ es una constante, $\mathrm{a}, \mathrm{b}$ y $\mathrm{c}$ representan fuentes de variación fijas e independientes, en este caso $a=a$ no $(1990=1, \ldots, 1997=8), b=$ meses $($ enero $=1, \ldots .$. diciembre $=12$ ), y $\mathrm{c}=$ período (abril-septiembre $=1$, octubre-marzo $=2$ ). Este último es un factor que toma en cuenta el período invernal y estival, y $\mathrm{CB}$ es la capacidad de bodega promedio por estratos de embarcación. Los estratos de embarcación fueron agrupados (Tabla 1), considerando la representatividad a lo largo de la serie de tiempo en base al número de naves, las capturas y esfuerzos de pesca. 
Tabla 1. Estratos de capacidad de bodega (CB) utilizados para el análisis de las tasas de captura, indicándose el número promedio de embarcaciones que operó en el período 1990-96 y la desviación estándar (d.e.).

Table 1. Hold capacity (HC) stratum as used to analyze the catch rates of the fleet. The average number of ships operating during the period 1990-96 and its standard deviation is also showed.

\begin{tabular}{|ccccr|}
\hline Estrato & $\begin{array}{c}\text { Rango de } \\
\mathbf{C B} \\
\mathbf{( m}^{3} \mathbf{)}\end{array}$ & $\begin{array}{c}\text { Marca de } \\
\text { Clase } \\
\left.\mathbf{( m}^{3}\right)\end{array}$ & $\begin{array}{c}\mathbf{N}^{\circ} \text { barcos } \\
\text { promedio }\end{array}$ & $\begin{array}{c}\text { d.e. } \\
(\mathbf{1 9 9 0 - 9 6 )}\end{array}$ \\
\hline 1 & $0-19$ & 10 & 4,3 & 2,82 \\
2 & $20-39$ & 30 & 10,7 & 7,15 \\
3 & $40-59$ & 50 & 41,2 & 15,41 \\
4 & $60-79$ & 70 & 20,0 & 7,81 \\
5 & $80-99$ & 90 & 7,5 & 4,42 \\
6 & $100-159$ & 130 & 7,9 & 3,97 \\
7 & $160-219$ & 190 & 2,5 & 1,65 \\
8 & $220-279$ & 250 & 6,1 & 3,25 \\
9 & $280-339$ & 310 & 2,0 & 1,08 \\
10 & $340-399$ & 370 & 5,4 & 2,77 \\
11 & $400-499$ & 450 & 3,0 & 1,66 \\
12 & $500-599$ & 550 & 2,1 & 1,04 \\
13 & $600-699$ & 650 & 2,4 & 2,98 \\
14 & $>700$ & 750 & 3,2 & 2,75 \\
\hline
\end{tabular}

La evaluación de las interacciones se realizó mediante una regresión paso a paso, utilizando $\mathrm{a}=0,05$. Los efectos año, mes y período no fueron logaritmizados, ya que sólo se consideran como indicadores de cambio entre un período de tiempo y otro.

Una vez analizada las tasas de captura, se seleccionó uno de los estratos de embarcación como estándar, considerando los siguientes requisitos: 1) participación permanente en la pesquería, al menos desde 1990, 2) cobertura espacial durante el desempeño de las operaciones de pesca, 3) características tecnológicas similares entre ellas, y 4) representatividad en la pesquería, tanto en número como en la magnitud de las capturas obtenidas.

\section{Abundancia relativa}

Una vez estandarizado el esfuerzo de pesca, el índice de abundancia más inmediato de los cambios en biomasa del stock corresponde a la captura por unidad de esfuerzo (CPUE), cuya estimación promedio ponderada se obtiene a partir de la relación entre la captura y el esfuerzo de pesca total estándar de las embarcaciones:

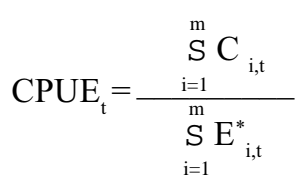

donde $\mathrm{C}_{\mathrm{i}, \mathrm{t}}$ es la captura (toneladas) del estrato de embarcaciones i en el mes $t$; $E_{i, t}^{*}$ es el esfuerzo de pesca estándar del estrato de embarcaciones i en el mes $\mathrm{t}, \mathrm{y} \mathrm{m}$ indica el número total de estratos.

\section{RESULTADOS}

\section{Tamaño de la flota y operación}

El número promedio de embarcaciones que han operado en la pesquería de $S$. bentincki y $E$. ringens, por estratos de $\mathrm{CB}$, indica que bajo los $80 \mathrm{~m}^{3}$ de $\mathrm{CB}$ se concentra el $76 \%$ de la flota en el período 1990 96, siendo el estrato de 40-59 $\mathrm{m}^{3}$ el que presenta la mayor frecuencia de participación con 39\% del promedio total (Tabla 1).

Anualmente han operado entre 223 y 261 embarcaciones, de las cuales entre $74 \%$ y $80 \%$ corresponde a embarcaciones menores a los $80 \mathrm{~m}^{3}$ de CB (Tabla 2). Sin embargo, este tamaño de flota no opera plenamente, ya que el número de embarcaciones

Tabla 2. Número de embarcaciones de la flota de cerco que ha operado en la pesquería de sardina común y anchoveta, con puerto base en la VIII Región.

Table 2. Annual number of purse-seines operating in the common sardine and anchovy fishery. Only ships with their main port located at the VIII Region of Chile.

\begin{tabular}{|cccc|}
\hline Año & $\begin{array}{c}\text { Flota } \\
<\mathbf{8 0} \mathbf{~ m}^{\mathbf{3}} \mathbf{C B}\end{array}$ & $\begin{array}{c}\text { Flota } \\
\mathbf{3} \mathbf{8 0 ~}^{\mathbf{3}} \mathbf{C B}\end{array}$ & TOTAL \\
\hline 1990 & 164 & 59 & 223 \\
1991 & 178 & 60 & 238 \\
1992 & 181 & 56 & 247 \\
1993 & 207 & 54 & 261 \\
1994 & 182 & 61 & 243 \\
1995 & 173 & 59 & 232 \\
1996 & 140 & 97 & 239 \\
\hline
\end{tabular}




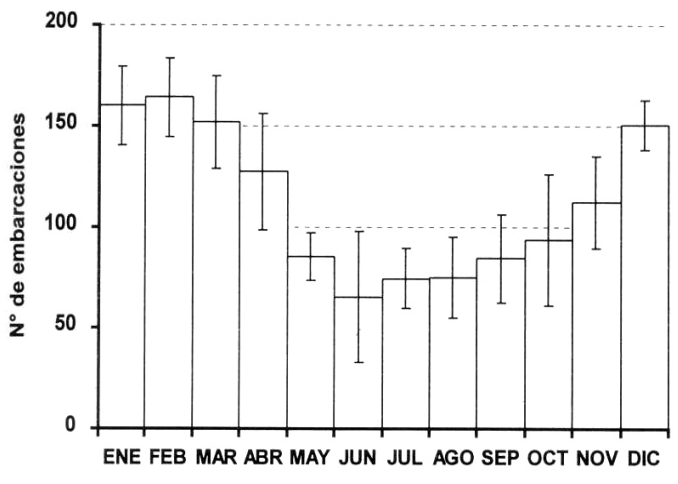

Figura 2. Número promedio de embarcaciones que operaron por mes en la pesquería de sardina común y anchoveta en el período 1990-96 ( \pm 1 desviación estándar).

Figure 2. Monthly average number of ships operating in the common sardine and anchovy fishery during the period 1990-96 ( \pm 1 standard desviation).

participantes exhibe un comportamiento estacional en la pesquería de $S$. bentincki y E. ringens. Entre diciembre y abril operan en promedio más de 100 embarcaciones por mes, reduciéndose a un rango entre 65 y 85 embarcaciones operando en la pesquería durante la época invernal (Fig. 2). Así, en el período 1990-96 el número promedio que operó en la pesquería alcanza solamente a 105 embarcaciones.

El análisis del régimen operacional, en términos de captura y viajes con pesca desarrollados, indica que el estrato de $40-59 \mathrm{~m}^{3}$ de $\mathrm{CB}$ es el que registra la mayor constancia entre 1990 y 1997, siguiendo en orden de importancia el estrato de $60-79 \mathrm{~m}^{3}$ (Fig. 3). La flota mayor a los $100 \mathrm{~m}^{3}$ de $\mathrm{CB}$ registra un reducido número de viajes con pesca anuales, pero las capturas obtenidas son muy similares al total de las menores de $80 \mathrm{~m}^{3}$ (Fig. 3).

\section{Tasas de captura}

Las tasas de captura anuales de cada embarcación se relacionan significativamente con la capacidad de bodega de las mismas (Tabla 3 ). Estas relaciones sólo se han establecido con las embarcaciones a las cuales efectivamente se les conoce la capacidad de bodega y que han operado más de un mes en la pesquería, constituyendo una fracción significativa del total (Tablas 2 y 3). De acuerdo con las relaciones
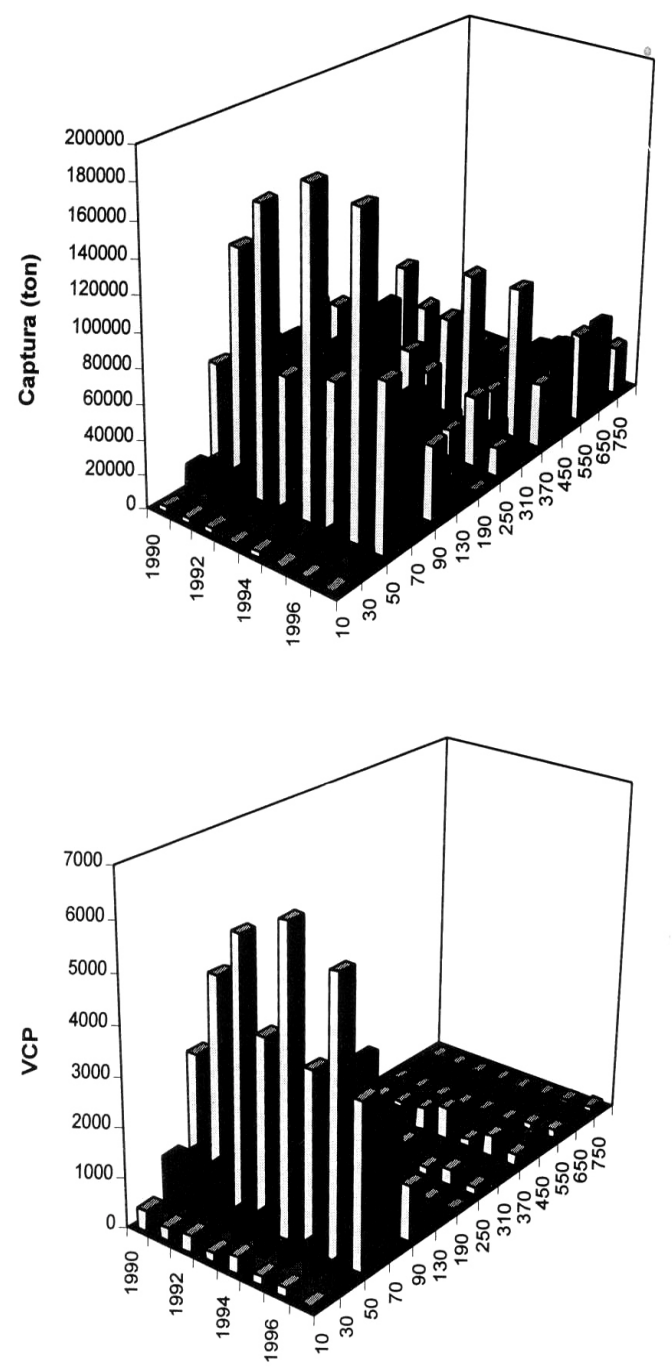

Figura 3. Capturas (arriba) y viajes con pesca (abajo) por año y estratos de capacidad de bodega de la flota. Período: enero de 1990-julio de 1997.

Figure 3. Annual catch (upper) and fishing trips (down) by hold capacity stratum of the fleet. Period: January 1990-July 1997.

encontradas, un análisis de covarianza que considera el año como fuente de variación, indica que al menos un año es significativamente diferente $(\mathrm{P}<0,05)$. Además, al aplicar una prueba de paralelismo se encuentran diferencias altamente significativas entre las pendientes $(\mathrm{F}=49,8 ;$ R.C. $=\{\mathrm{F} \mid \mathrm{F}>$ $2,57$ v $\mathrm{F}<0,17\} ; \mathrm{a}=0,05)$; mientras que una prueba de concurrencia para averiguar si las curvas se ori- 
Tabla 3. Coeficientes de las relaciones anuales entre el logaritmo de las tasas de captura y el logaritmo de la capacidad de bodega de la flota de cerco que operó sobre $S$. bentincki y $E$. ringens en el área de Talcahuano en el período 1990-96. In $(\mathrm{CB})=$ logaritmo de la capacidad de bodega, $\pm \mathrm{LC}=$ límites de confianza de $95 \%, \mathrm{~N}=$ número de barcos, $\mathbf{R}^{2}=$ coeficiente de determinación.

Table 3. Coefficients of the annual relationships obtained between the log-transformed catch rates and the logtransformed hold capacity of the seines operating in the common sardine and anchovy fishery in the area off Talcahuano between 1990-96. Nomenclature: $\ln (C B)=$ log-transformed hold capacity, \pm LC $=95 \%$ confidence limits, $\mathbf{N}=$ number of ships, $\mathbf{R}^{2}=$ coefficient of determination.

\begin{tabular}{|cccccc|}
\hline \multicolumn{7}{c|}{ Coeficientes de regresión } \\
Ã̃NO & Intercepto $( \pm \mathbf{L C})$ & $\mathbf{L n}(\mathbf{C B})( \pm \mathbf{L C})$ & $\mathbf{N}$ & $\mathbf{R}^{\mathbf{2}}$ & Error típico \\
\hline 1990 & $-0,356( \pm 0,303)$ & $0,891( \pm 0,073)$ & 147 & 0,800 & 0,419 \\
1991 & $-0,191( \pm 0,282)$ & $0,894( \pm 0,066)$ & 180 & 0,800 & 0,410 \\
1992 & $+0,364( \pm 0,254)$ & $0,735( \pm 0,060)$ & 175 & 0,774 & 0,337 \\
1993 & $-0,538( \pm 0,377)$ & $0,841( \pm 0,087)$ & 178 & 0,673 & 0,507 \\
1994 & $+0,037( \pm 0,241)$ & $0,807( \pm 0,055)$ & 179 & 0,828 & 0,335 \\
1995 & $-0,849( \pm 0,308)$ & $0,980( \pm 0,069)$ & 169 & 0,824 & 0,419 \\
1996 & $+0,063( \pm 0,212)$ & $0,842( \pm 0,046)$ & 203 & 0,864 & 0,333 \\
\hline
\end{tabular}

ginan en un mismo punto en la ordenada también revela diferencias significativas $(\mathrm{F}=6,47 ; \mathrm{R} . \mathrm{C} .=\{\mathrm{F} \mid \mathrm{F}$ $>2,57$ v F $<0,17\} ; a=0,05)$.

Aun cuando la capacidad de bodega explica una fracción significativa de la variabilidad observada en las tasas de captura anuales de las embarcaciones, ciertos factores que permanecen fijos dentro del ciclo anual e interanualmente podrían generar diferencias en las tasas de captura. En este contexto, el análisis con un modelo lineal generalizado debería otorgar una mejor respuesta en términos del valor promedio esperado de las tasas de captura mensuales. Previo a dicho análisis, se encontró la existencia de 20 puntos atípicos (outliers) los cuales fueron descartados de análisis posteriores, representando una remoción del 2\% de la información disponible. Las tasas de captura transformada a logaritmo (ln(cpue)) revela que de 11 efectos de entrada a la regresión paso a paso, sólo 5 resultan estadísticamente significativos, siendo tres de ellos interacciones entre los factores. El modelo final es altamente significativo $\left(r^{2}=0,840 ; p<0,001\right)$ y queda expresado por:

$$
\begin{aligned}
\ln (R)= & 0,220+0,808 \cdot C B+0,01 \cdot C B \cdot a \cdot b \cdot c \\
& -0,05 \cdot a \cdot b \cdot c+0,021 \cdot b \cdot c-0,078 \cdot b
\end{aligned}
$$

donde a es el factor asociado al año, b al mes y c al período, en tanto CB es la capacidad de bodega promedio de las embarcaciones en cada estrato.

\section{Esfuerzo de pesca}

El análisis del régimen operacional de la flota revela que el estrato de embarcaciones de $40-59 \mathrm{~m}^{3} \mathrm{de}$ $\mathrm{CB}$ cumple con algunos de los requisitos para ser seleccionado como categoría estándar de embarcaciones en esta pesquería (Fig. 3). La razón entre el rendimiento estimado con la ecuación (6) para los estratos de embarcaciones i y el rendimiento estimado para el estrato estándar permite corregir el esfuerzo de pesca nominal observado. Dicha estandarización revela un aumento de los viajes con pesca, lo que resulta en una disminución de la CPUE (Fig. 4). El aumento de los viajes con pesca estándar ocurre principalmente debido al mayor poder de pesca relativo de las embarcaciones mayores a 80 $\mathrm{m}^{3}$ de $\mathrm{CB}$, las cuales tienen una participación más activa durante la época estival de cada año. En efecto, al analizar independientemente las capturas, esfuerzo y CPUE entre dos grupos de embarcaciones, uno menor a $80 \mathrm{~m}^{3}$ de $\mathrm{CB}$ y otro mayor a dicho límite, indica que los niveles de capturas son similares entre ambos grupos (Fig. 5). No obstante, la flota ${ }^{3} 80 \mathrm{~m}^{3}$ realiza un número mucho menor de viajes con pesca, lo que resulta en niveles de rendimiento nominal mucho mayores para esta flota (Fig. 6). Sin embargo, al estandarizar el esfuerzo de pes- 

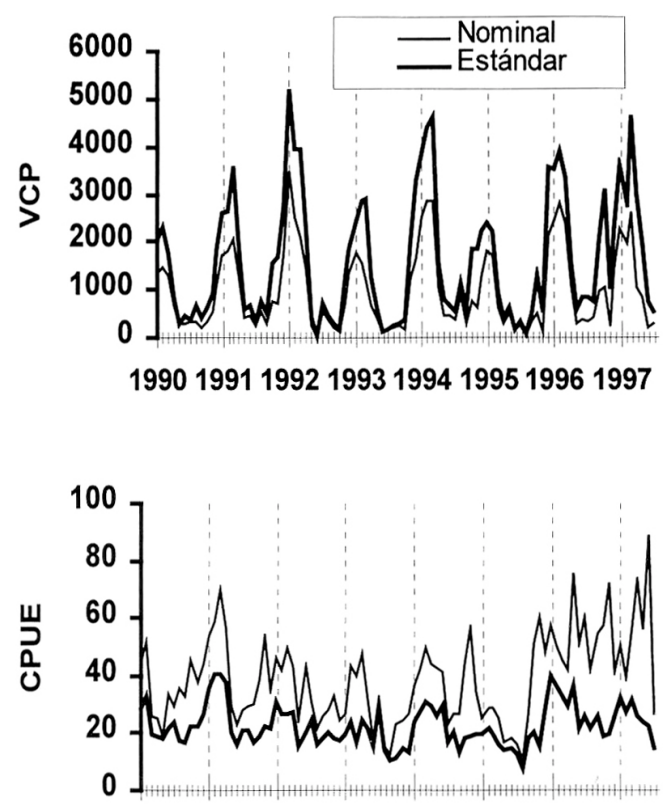

19901991199219931994199519961997

Figura 4. Viajes con pesca (arriba) y tasas de captura (abajo), nominal y estándar para la pesquería de sardina común y anchoveta en el período enero de 1990 y julio de 1997.

Figure 4. Nominal and standard fishing trips (upper) and catch rates (down) for the common sardine and anchovy fishery during the period January 1990-July 1997.

ca se observa que las tasas de captura de estos dos grupos de embarcaciones llegan a ser similares (Figs. 5 y 6 ), lo que permite deducir que el mayor rendimiento nominal observado para la flota $380 \mathrm{~m}^{3}$ responde exclusivamente al mayor tamaño de sus embarcaciones (Tabla 3).

Estacionalmente, la flota ejerce en promedio sobre 3000 viajes con pesca estándar en el primer trimestre para disminuir en abril aproximadamente a la mitad. Entre mayo y octubre, la flota realiza un menor esfuerzo de pesca, particularmente porque la flota industrial de mayor tamaño orienta su esfuerzo de pesca hacia T. s. murphyi. Las embarcaciones artesanales salen de la pesquería en abrilmayo por razones de seguridad en la navegación debido a las condiciones climáticas adversas que comienzan a presentarse a contar de esos meses. Por el contrario, cuando las condiciones climáticas comienzan a mejorar en octubre, el esfuerzo de pesca vuelve nuevamente a incrementarse (Fig. 7).
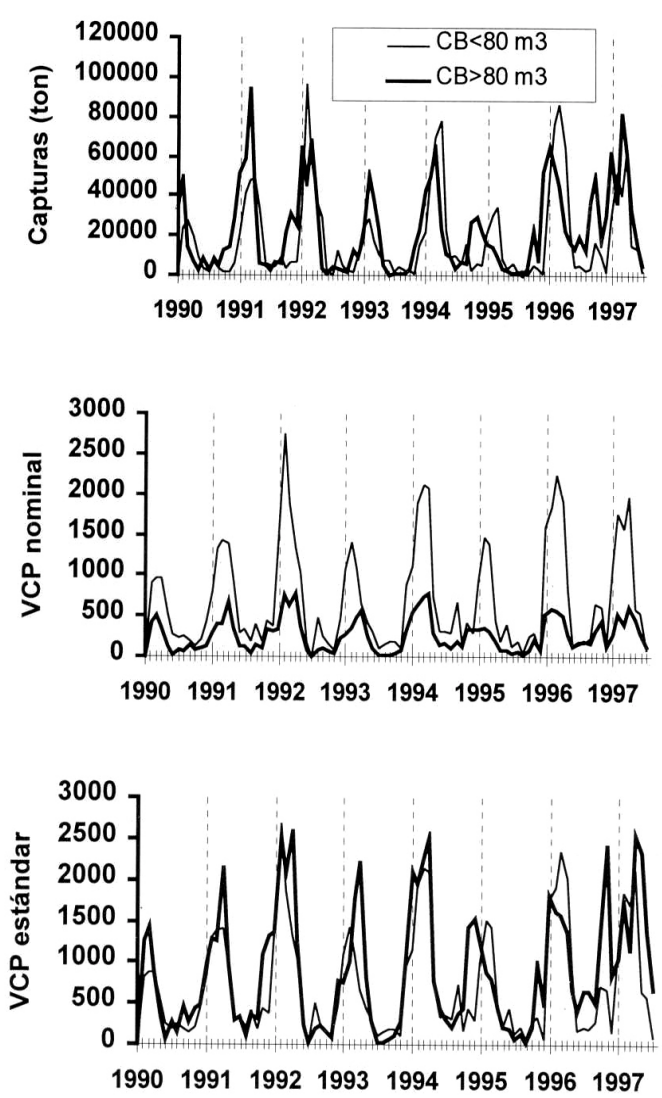

Figura 5. Captura y esfuerzo de pesca (VCP), nominal y estándar para dos grupos de embarcaciones de la flota de cerco que opera en la pesquería de sardina común y anchoveta (1990-97).

Figure 5. Catch and nominal and standard fishing trips (VCP) for two groups of ships operating in the common sardine and anchovy fishery (1990-97).

\section{Abundancia relativa}

La captura por unidad de esfuerzo estándar (CPUE), expresada en toneladas por viajes con pesca estándar, se considera un índice de abundancia relativa. La CPUE de S. bentincki ha declinado persistentemente desde comienzos de 1991 hasta julio de 1995, recuperándose levemente hacia fines de 1995 y comienzos de 1996. En el caso de E. ringens, el índice de abundancia se mantuvo relativamente estable entre 1990 y 1992, aunque con fluctuaciones estacionales. Luego, a mediados de 1993 y comienzos de 1994 la CPUE de anchoveta se incrementa notablemente para posteriormente exhibir una tendencia decreciente hasta fines de 1995; luego, en 1996-97, la 

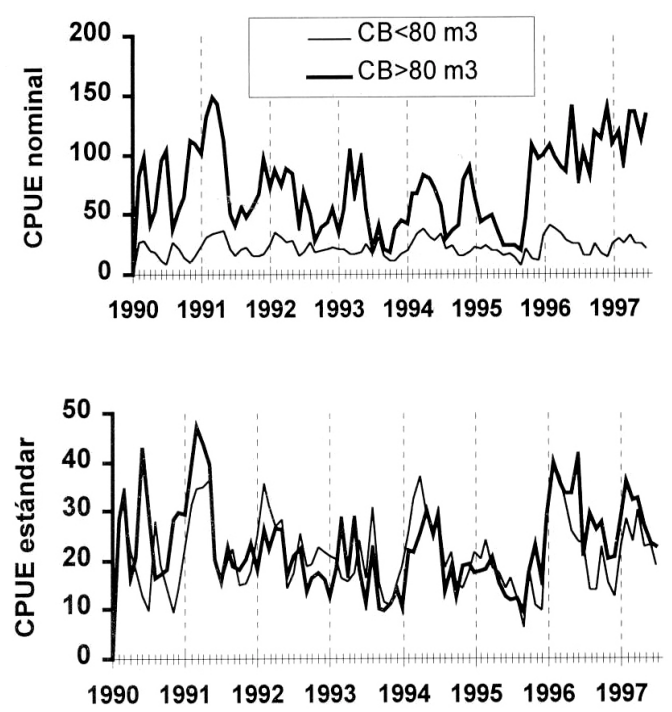

Figura 6. Tasas de captura (CPUE), nominal y estándar, para dos grupos de embarcaciones de la flota de cerco que opera en la pesquería de sardina común y anchoveta (1990-97).

Figure 6. Nominal and standard catch rates (CPUE) for two groups of ships operating in the common sardine and anchovy fishery (1990-97).

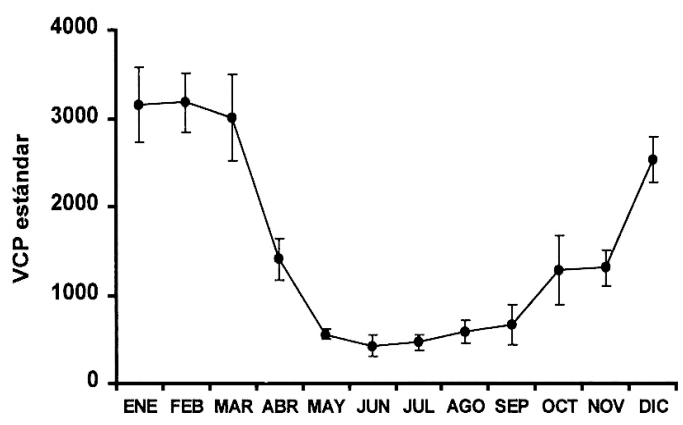

Figura 7. Comportamiento estacional de los viajes con pesca estándar en la pesquería de sardina común y anchoveta (1990-96). Las barras verticales representan un error estándar.

Figure 7. Seasonal behaviour of the standard fishing trips for the common sardine and anchovy fishery (1990-96). The vertical bar is one standard error.
CPUE de anchoveta se estabiliza en niveles promedio similar a los observados en 1990-92 (Fig. 8).

En ambas especies se reconoce un componente de cambio estacional asociado a la mayor disponibilidad de estos recursos en la zona costera, particularmente durante la época estival en el caso de $S$. bentincki y hacia otoño-invierno en el caso de $E$. ringens, lo que se observa mejor a través de la señal anual de la abundancia relativa de ambos recursos (Fig. 9).

Anualmente, mientras la CPUE de $S$. bentincki manifiesta una tendencia decreciente desde 1991 a 1995, la CPUE de E. ringens exhibe una tendencia creciente entre 1992 y 1994, para luego comenzar a disminuir persistentemente en concomitancia con una recuperación de la abundancia relativa de $S$. bentincki en 1996.

\section{DISCUSION}

Al utilizar los datos de captura y esfuerzo de pesca para estimar cambios en la abundancia de los stocks de peces se puede encontrar una gran variedad de problemas (Gulland, 1974). Esos problemas se refieren a la cobertura de los datos sobre la extensión espacial de distribución de los stocks y si estos datos, que provienen de una pesquería que intenta mantener tasas de captura altas, reflejan realmente la abundancia del stock. De particular importancia en pesquerías pelágicas de peces que forman agregaciones y cardúmenes es la dependencia que puede exhibir el coeficiente de capturabilidad respecto del tamaño del stock (Csirke, 1988; 1989; Patterson et al., 1993). Los efectos de la denso dependencia del coeficiente de capturabilidad ocurren particularmente cuando el tamaño del stock está bastante disminuido y cuando la intensidad de explotación es alta. Este fenómeno ha sido documentado por Csirke (1989) para el stock centro-norte de E. ringens en Perú, y se podría inferir para los clupeiformes de Talcahuano. Sin embargo, hasta no contar con estimaciones independientes del tamaño de los stocks, el uso de la CPUE como índice de abundancia puede seguir siendo útil para documentar los cambios relativos en la abundancia y la disponibilidad de estas especies. Se destaca que la problemática de la denso-dependecia del coeficiente de capturabilidad ha sido propuesto para $S$. bentincki por Veloso y Arrizaga (1987), pero estos autores no describen adecuadamente la metodología ni las estimaciones de la abundancia absoluta del stock. Por 

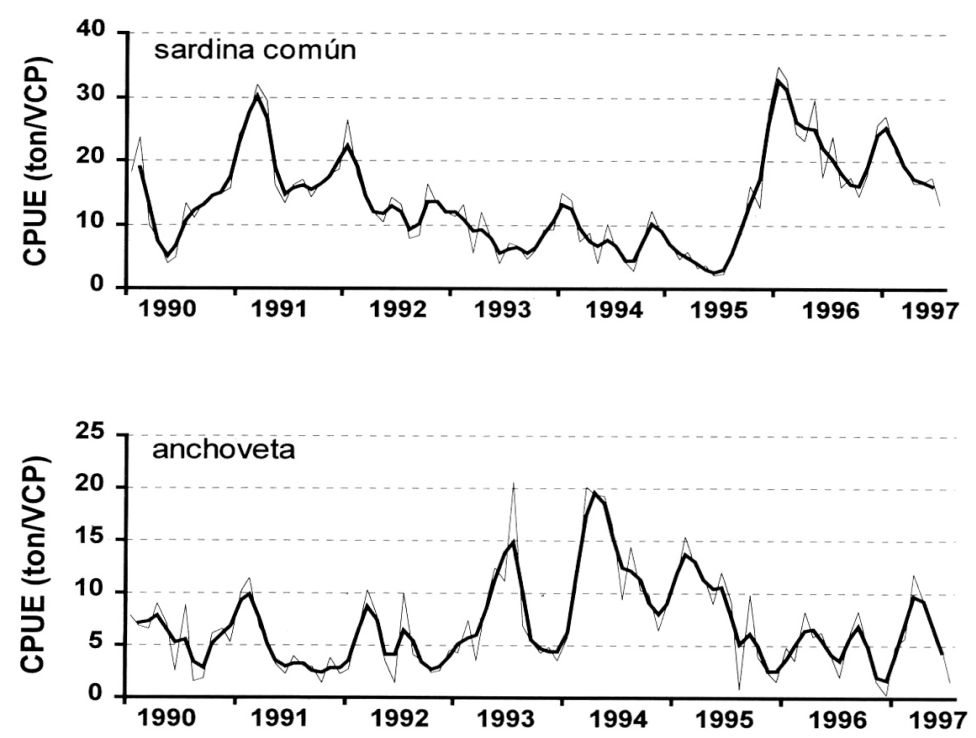

Figura 8. Captura por unidad de esfuerzo estándar (CPUE) de sardina común y anchoveta en el período enero de 1990-julio de 1997. Las líneas gruesas corresponden a un suavizamiento de la serie original (líneas delgadas).

Figure 8. Catch per unit effort (CPUE) of common sardine (upper) and anchovy (down) during the period January 1990-July 1997. The thick lines are a smoothed of the original series (thin lines).

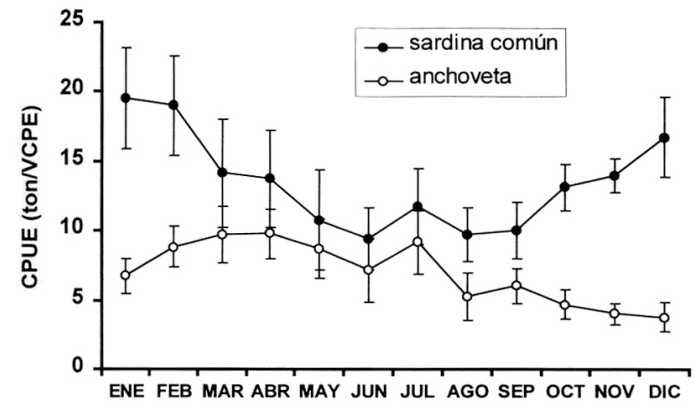

Figura 9. Comportamiento estacional de la abundancia relativa (CPUE) de sardina común y anchoveta en el período 1990-97. Las barras verticales indican un error estándar.

Figure 9. Seasonal behaviour of the catch per unit effort (CPUE) of common sardine and anchovy during the period 1990-97. The vertical bar is one standard error.

mientras, se debe considerar que el coeficiente de capturabilidad no presenta cambios significativos asociados al tamaño del stock durante la serie de tiempo analizada.

Por otra parte, varios otros factores asociados a cambios en los aspectos operacionales y tecnológicos de la flota podrían estar influyendo en las tasas de captura. En la pesquería de sardina común y anchoveta se observa que en términos de unidades de pesca la flota más importante se encuentra bajo los $80 \mathrm{~m}^{3}$ de capacidad de bodega. Varias de estas embarcaciones caen en la categoría de lanchas artesanales, las cuales son propiedad de pequeños armadores, lo que impide llevar un registro sistemático de los cambios técnico-operacionales. No obstante, en este estudio queda demostrado que el poder de pesca de las embarcaciones depende de su tamaño. En efecto, las fluctuaciones en las tasas de captura estándar de dos categorías de la flota, separadas por el límite de los $80 \mathrm{~m}^{3}$, son muy similares e indica que la remoción de los efectos a través de la estandarización podría dar cuenta de un índice de abundancia que refleje los cambios que están ocurriendo en el tamaño del stock de $S$. bentincki y $E$. ringens en la zona centro-sur de Chile.

El poder de pesca depende del tamaño de la embarcación, en términos de la capacidad de bodega, y de la época del año. Resultados similares encuentran Caballero et al. (1992) para el análisis del poder de pesca en las pesquerías de Sardinops sagax de la zona norte (Arica-Coquimbo) y de T. s. murphyi de la zona centro-sur (Talcahuano) y por Böhm et al. (1996). Para este trabajo las únicas variables dis- 
ponibles fueron la capacidad de bodega y los viajes con pesca, restricción impuesta por el acceso a la información de la flota menor a $80 \mathrm{~m}^{3}$ principalmente. Esta limitante no permitió indagar en la influencia de otras variables funcionales y operacionales que podrían estar explicando el poder de pesca de las embarcaciones de la flota. Sin embargo, tal como ha sido encontrado por Caballero et al. (1992) y Böhm et al. (1996), la capacidad de bodega y los viajes con pesca son las variable que mejor explican los cambios en las capturas de las embarcaciones; resultados similares encuentra Yáñez et al. (1993) para la pesquería de anchoveta y sardina en la zona norte de Chile.

En relación con la estacionalidad de las capturas de $S$. bentincki y E. ringens, se está de acuerdo con Cubillos y Arancibia (1993a, b) en que dicho comportamiento se debe a tres factores principales: 1) las mejores condiciones climáticas de la época estival, lo que favorece a la operación de embarcaciones menores, 2) el reclutamiento de $S$. bentincki ocurre entre noviembre y diciembre, lo que incide en un aumento de la abundancia y disponibilidad (cardúmenes y agregaciones de alta densidad en zonas costeras), y 3 ) reorientación en primavera del esfuerzo de pesca de algunas embarcaciones industriales, que capturan principalmente $T$. s. murphyi el resto del año (otoño-invierno).

El comportamiento estacional de las capturas, por lo tanto, se corresponde con el comportamiento del esfuerzo de pesca y con la abundancia relativa de $S$. bentincki y E. ringens. Al respecto, en el esfuerzo de pesca estándar no se aprecian cambios en el promedio ni en la varianza de la serie, lo que permite plantear que el esfuerzo de pesca de la flota se encuentra relativamente estable en el período analizado.

En relación con los cambios estacionales de la abundancia relativa de $S$. bentincki, la captura por unidad de esfuerzo estándar (CPUE) es máxima entre noviembre y abril. Se destaca que la principal época reproductiva de $S$. bentincki ocurre entre julio y septiembre (Cubillos y Arancibia, 1993a, b; Arancibia et al., 1994), con un máximo de abundancia larval en septiembre-octubre (Sepúlveda, 1990). Esta situación se traduce en un máximo de abundancia de juveniles en primavera-verano y, por ende, con la época de reclutamiento entre noviembre y diciembre. En efecto, en estos meses en las capturas de la flota ocurre una alta proporción de

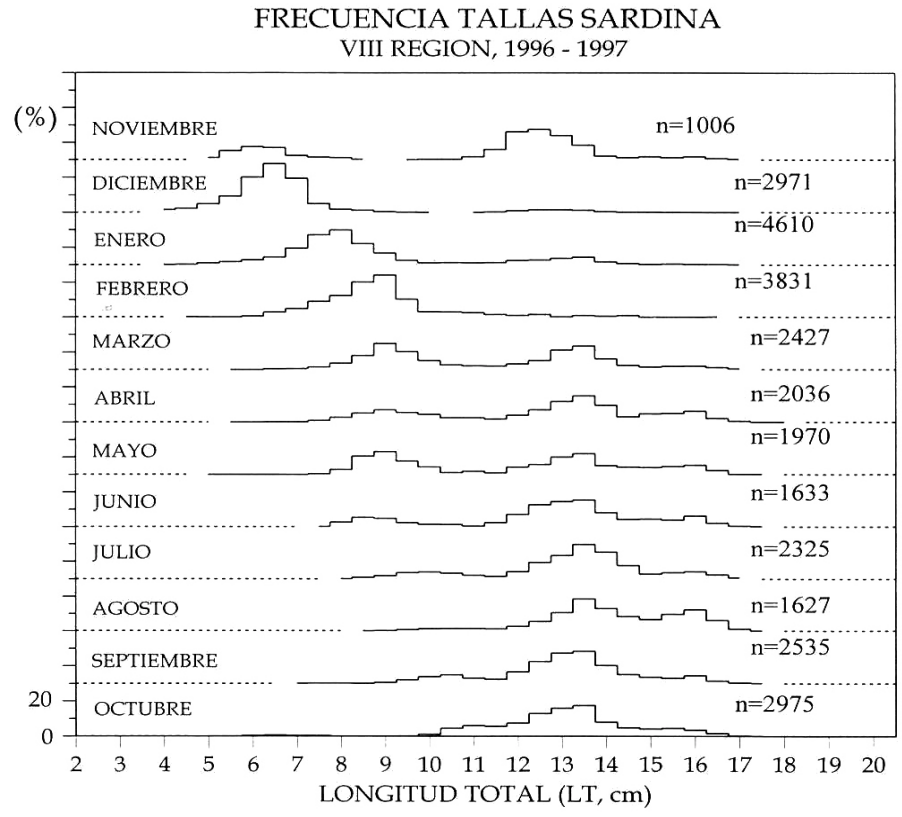

Figura 10. Evolución mensual de las frecuencias de tamaños de sardina común entre noviembre de 1996 y octubre de 1997, donde se aprecia el reclutamiento de la clase anual de 1996 en noviembre del mismo año.

Figure 10. Monthly common sardine length frecuency data between November 1996 to October 1997. Recruitment of the 1996 year class is observed in November 1996. 
ejemplares juveniles de tamaño modal de 6 a $7 \mathrm{~cm}$ de longitud total (Fig. 10). La fracción juvenil del stock de $S$. bentincki que se recluta en esa época, sin duda es altamente abundante y por lo tanto fácilmente detectable por la flota, más aún si se acepta que se distribuye a la forma de cardúmenes y agregaciones en zonas costeras protegidas, como la Bahía de Concepción y el Golfo de Arauco. En consecuencia, la abundancia de $S$. bentincki es máxima en la época estival debido a que en dicha época ocurre el reclutamiento, situación que determina el comportamiento estacional de la pesquería, traduciéndose en una pesquería cuyos rendimientos son dependientes del reclutamiento (Cubillos y Arancibia, 1993a, b).

Por otra parte, los cambios interanuales de la CPUE indican que la abundancia de $S$. bentincki presentó una tendencia decreciente desde comienzos de 1991 hasta mediados de 1995, recuperándose en 1996 y 1997. En tanto, la abundancia de $E$. ringens fue mayor hacia fines de 1994 y parte de 1995. Estas tendencias relativamente opuestas en la abundancia de ambas especies se podría deber a varias causas (MacCall, 1983), entre ellas una probable competencia interespecífica. No obstante, se debe tener en cuenta que la hipótesis de competencia es difícil de demostrar, sobre todo debido a la corta serie de tiempo que en este trabajo se analiza. En todo caso, se debe tener presente que la hipótesis de competencia o de reemplazo ha sido planteada en más de una oportunidad cuando se analiza la sustitución recíproca en el rol de especies dominantes del llamado complejo "sardina-anchoveta" (Daan, 1980; Pauly, 1987; Serra y Tsukayama, 1988), así como para otras especies de clupeiformes (Cury y Fontana, 1988).

\section{AGRADECIMIENTOS}

Parte de los resultados de este estudio han sido obtenidos en el marco de los Proyectos FIP N ${ }^{\circ}$ 94-11: Evaluación de la pesquería y del stock de sardina común en la zona centro-sur, y FIP $N^{\circ}$ 96-10: Evaluación indirecta del stock de anchoveta y sardina común en la zona centro-sur. Los autores agradecen al Consejo de Investigación Pesquera las facilidades otorgadas para publicar parte de los resultados de dichos estudios. También se agradece a los tres revisores anónimos del manuscrito, cuyas sugerencias y comentarios permitieron mejorar varios aspectos del mismo.

\section{REFERENCIAS}

Allen, R.L. y R.G. Punsly. 1984. Catch rates as indices of abundance of yellowfin tuna (Thunnus albacares) in the Eastern Pacific Ocean. Bull. Inter-Amer. Trop. Tuna Comm. 18 (4): 301-379.

Arancibia, H., R. Alarcón, L. Cubillos y J. Remaggi. 1994. Determinación de la talla de primera madurez sexual y fecundidad parcial en la sardina común, Strangomera bentincki (Norman, 1936), del área de Talcahuano, Chile. Biología Pesquera 23: 11-17.

Böhm, G., M.A. Barbieri, E. Yáñez, V. Catasti y A. González. 1996. Análisis de la captura y del esfuerzo de pesca de las unidades de pesquería de jurel de la zona centro-sur y norte. Informe Final Proyecto FIP N ${ }^{\circ} 25 / 94$. Est. y Doc., Escuela de Ciencias del Mar, UCV, Valparaíso, 133 p.

Caballero, L., L. Santillán y G. Rosson. 1992. Investigación del esfuerzo pesquero en las principales pesquerías pelágicas chilenas: sardina española Zona Norte y jurel Talcahuano. CORFO-IFOP, SGI-IFOP 92/16, Inst. Fom. Pesq., Valparaíso, Chile, 52 p.

Carlson, C. 1975. The measurement of relative fishing power using cross section production functions. Rapp. P-v. Int. Explor. Mer 168: 84-98.

Csirke, J. 1988. Small shoaling pelagic fish stocks. In: Fish population dynamics, J.A. Gulland (Ed.), John Wiley y Sons., New York, 271-302.

Csirke, J. 1989. Changes in the catchability coefficient in the peruvian anchoveta (Engraulis ringens) fishery. In: The peruvian upwelling ecosystem: dynamics and interactions. D. Pauly, P. Muck, J. Mendo e I. Tsukayama (Eds.), ICLARM Contribution $\mathrm{N}^{\circ} 18,207-219$.

Cubillos, L. y H. Arancibia. 1993a. Análisis de la pesquería de sardina común y anchoveta del área de Talcahuano: situación actual y perspectivas. Doc. Téc. Inst. Invest. Pesq., Talcahuano, Chile, 2 (2): 1-19.

Cubillos, L. y H. Arancibia. 1993b. Análisis de la pesquería de sardina común (Strangomera bentincki) y anchoveta (Engraulis ringens) del área de Talcahuano, Chile. Invest. Mar., Valparaíso, 21: 3-21.

Cury, P. y A. Fontana. 1988. Compétition et stratégies démographiques comparées de deux espèces de sardinelles (Sardinella aurita et Sardinella maderensis) des côtes ouest-africaines. Aquat. Living Resour. 1: 165-180. 
Daan, N. 1980. A review of replacement of depleted stocks by other species and the mechanisms underlying such replacement. Rapp. P-v. Reun. Cons. Int. Explor. Mer. 177: 405-421.

Fonseca, T., E. Yáñez y O. Barra. 1986. Relación entre la temperatura superficial del mar y capturas comerciales en el área de Talcahuano (1965-1976). In: La Pesca en Chile. P. Arana (Ed.), Escuela de Ciencias del Mar, UCV, Valparaíso, 243-248.

Guerrero, A. y E. Yáñez. 1986. Análisis de las principales pesquerías pelágicas desarrolladas en la zona de Talcahuano $\left(37^{\circ} \mathrm{S}-73^{\circ} \mathrm{W}\right)$ entre 1965 y 1984. In: La Pesca en Chile. P. Arana (Ed.), Escuela de Ciencias del Mar, UCV, Valparaíso, 223-241.

Gulland, J.A. 1974. Catch per unit effort as a measure of abundance. Inter. Comm. Cons. Atlan. Tunas, Coll. Vol. Sci. Pap. 3: 1-5.

Hilborn, R. y C.J. Walters. 1992. Quantitative fisheries stock assessment: choice, dyanamics and uncertainty. Chapman and Hall, New York, USA, 570 p.

Kimura, D.K. 1981. Standardized measure of relative abundance based on modelling log (CPUE), and their application to Pacific Ocean perch (Sebastes alutus). J. Cons. int. Explorer. Mer. 39: 211-218.

MacCall, A.D. 1983. Informe del grupo de trabajo sobre investigación y monitoreo de recursos. En: Informes de la consulta de expertos para examinar los cambios en la abundancia y composición por especies de recursos de peces neríticos. FAO Inf. Pesca, (291) 1: 9-42.

Patterson, K., T. Pitcher y T. Stokes. 1993. A stock collapse in a fluctuating enviroment: the chub mackerel Scomber japonicus in the eastern central Pacific. Fish. Res. 18: 199-218.

Pauly, D. 1987. Managing the Peruvian upwelling ecosystem: a synthesis. In: The Peruvian anchoveta and its upwelling ecosystem: three decades of changes. D. Pauly y I. Tsukayama (Eds.), ICLARM Contribution $\mathrm{N}^{\circ} 391,325-342$.

Sepúlveda, A. 1990. Variabilidad temporal del ictioplancton en un área de surgencia costera de Chile central: procesos ambientales y biológicos asociados. Tesis Magister en Ciencias, Oceanografía, Universidad de Concepción, Chile, 81 p.
Serra, J.R. 1978. La pesquería de sardina común (Clupea (Strangomera) bentincki) y anchoveta (Engraulis ringens) de Talcahuano: análisis de su desarrollo y situación actual. Invest. Pesq. (Chile), 29: 1-21.

Serra, J.R. 1983. Changes in the abundance of pelagic resources along the Chilean coast. In: Actas de la Consulta de Expertos para examinar los cambios en la abundancia y composición por especies de recursos neríticos. G.D. Sharp y J. Csirke (Eds.). FAO, Inf. Pesca 291, Vol. 2, 255-284.

Serra, J. y I. Tsukayama. 1988. Sinópsis de datos biológicos y pesqueros de la sardina, Sardinops sagax, en el Pacífico suroriental. FAO. Sinop. Pesca (13) Rev. 1:60 p.

Shimada, B. y M. Schaefer. 1956. A study of changes in fishing effort, abundance, and yield for yellowfin and skipjack tuna in the Eastern Tropical Pacific Ocean. Bull. Inter-Amer. Trop. Tuna Comm. 1: 347-421.

Veloso, C. y A. Arrizaga. 1987. El esfuerzo de pesca de la flota cerquera en la zona de Talcahuano (1965-1985). Una revisión preliminar del problema. In: Manejo y desarrollo pesquero. P. Arana (Ed.), Escuela de Ciencias del Mar, UCV, Valparaíso, Chile, 167-179.

Yáñez, E. y L. Maritano. 1983. Análisis histórico (1971-1980) de la pesquería pelágica de la zona norte de Chile ( $\left.18^{\circ} 20^{\prime} \mathrm{S}-24^{\circ} 00^{\prime} \mathrm{S}\right)$ y estimación del estado de los recursos explotados. In: Análisis de Pesquerías Chilenas. P. Arana (Ed.), Escuela de Ciencias del Mar, UCV, Valparaíso, Chile, 161-177.

Yáñez, E., M.A. Barbieri y A. Montecinos. 1990. Relaciones entre las variaciones del medio ambiente y las fluctuaciones de los principales recursos pelágicos explotados en la zona de Talcahuano, Chile. In: Perspectivas de la Actividad Pesquera en Chile. M.A. Barbieri (Ed.), Escuela de Ciencias del Mar, UCV, Valparaíso, Chile, 49-62.

Yáñez, E., M.A. Barbieri y L. Santillán. 1992. Longterm environmental variability and pelagic fisheries in Talcahuano, Chile. In: The Benguela Trophic Functioning. A.I.L. Payne, K.H. Mann y R. Hilborn (Eds.), S. Afr. J. Mar. Sci. 12: 175-188.

Yáñez, E., C. Canales, M.A. Barbieri, A. González y V. Catasti. 1993. Estandarización del esfuerzo de pesca y distribución espacial e interanual de la CPUE de anchoveta y sardina en la zona norte de Chile entre 1987-1992. Invest. Mar, Valparaíso, 21: 111-132. 